\title{
Crossability between Transgenic Blue Chrysanthemums and the Wild Chrysanthemum Species Chrysanthemum japonense var. japonense
}

\author{
Ryutaro AIDA ${ }^{1 *}$, Naonobu NODA ${ }^{1}$, Satoshi YOSHIOKA ${ }^{1}$, \\ Mitsuru DOUZONO ${ }^{1}$, Mai TSUDA ${ }^{2}$ and Ryo OHSAWA ${ }^{2}$ \\ ${ }^{1}$ Institute of Vegetable and Floriculture Science, National Agriculture and Food Research \\ Organization, Tsukuba, Japan \\ ${ }^{2}$ Gene Research Center, Tsukuba Plant Innovation Research Center, University of Tsukuba, Tsukuba, \\ Japan
}

\begin{abstract}
Transgenic chrysanthemums (Chrysanthemum morifolium) with blue flower colors have already been created, and are expected to be commercialized. However, cultivated chrysanthemums are known to cross with wild species native to Japan, and careful studies are needed to assess the risk of these transgenes escaping into wild Chrysanthemum populations. We previously reported on the transmission of transgenes from the model cultivar 'Taihei' to interspecific progeny. For this study, we used the recently developed cultivar 'Sei Arabella' and a promising breeding strain (T37) as transgene hosts, and performed crosses between these lines and the wild species Chrysanthemum japonense var. japonense. We found relatively high seed set rates (20.6\%-83.4\%) after artificial, reciprocal pollinations, and the inheritance and segregation of the transgenes in the hybrid progeny were confirmed by PCR. Some of the transgenic progeny exhibited blue flower colors and contained modified anthocyanins, like their transgenic parents. These results were similar to those obtained with 'Taihei' and thus suggested that the risk of transgenes escaping to wild species could be quite high. Therefore, it will be important to investigate techniques to reduce this risk.
\end{abstract}

Discipline: Horticulture

Additional key words: environmental risk, flower color, ornamental plants, transformation

\section{Introduction}

Chrysanthemum is one of the world's most economically important ornamental plants, especially in Japan, and the creation of novel flower colors is an important goal in this industry. There are various flower colors available in chrysanthemums, although bluish ones have only been created recently. Violet-colored chrysanthemums were produced using transgenic techniques involving anthocyanin B-ring hydroxylation (Brugliera et al. 2013, Noda et al. 2013). This strategy was also employed to produce bluish roses (Katsumoto et al. 2007) and bluish carnations (Tanaka et al. 1998). Truly blue chrysanthemums were then produced by combining the same process with glucosylation (Noda et al. 2017). This new flower color is expected to generate new consumer demand and increase chrysanthemum production. Bluish roses and carnations have now been commercialized, and chrysanthemums will follow.

Ohashi \& Yonekura (2004) reported that there are 32 species of Chrysanthemum in Japan, many of which can be crossed with chrysanthemum cultivars (Nakata et al. 1987, Nakata et al. 2001, Ohashi \& Yonekura 2004). Therefore, the risk associated with crossing between wild varieties and genetically modified chrysanthemums must be carefully assessed before the genetically modified chrysanthemums can be commercialized. We have previously reported on the transmission of transgenes from blue chrysanthemums produced using the model host cultivar 'Taihei' to interspecific progeny (Aida et al. 2018).

For this study, we selected the recently developed cultivar 'Sei Arabella' and the promising breeding line T37 for use as transgenic hosts. As these lines will be 
used as hosts for the commercialization of blue chrysanthemums, we examine the crossability between these chrysanthemums and the wild species Chrysanthemum japonense var. japonense. As we found with 'Taihei', the transgenes were inherited by the hybrid progeny, suggesting a possibly high risk of transgenes escaping from cultivated chrysanthemums into wild Chrysanthemum populations.

\section{Materials and methods}

\section{Plants}

The blue transgenic lines 2081-33 and 2013-03 were derived from the chrysanthemum cultivar 'Sei Arabella' and the breeding line T37, respectively. Both lines were transformed using the binary vector pB423 (Noda et al. 2017), which includes a flavonoid 3',5'-hydroxylase $\left(F 3^{\prime} 5^{\prime} H\right)$ gene from Campanula medium (GenBank Accession No. FW570877) and the $A 3^{\prime} 5^{\prime} G T$ gene (AB115560) from Clitoria ternatea, which encodes a UDP-glucose:anthocyanin 3',5'-O-glucosyltransferase.

Wild-type 'Sei Arabella' and T37 plants were used as controls. A laboratory line (GU3) of Chrysanthemum japonense var.japonense, which is a wild Chrysanthemum species native to Japan, was used as one parent in crossing experiments. This species was selected because it is a common wild Chrysanthemum species having the same hexaploid chromosome set as those of commercially produced chrysanthemums.

\section{Crossing and seed germination experiments}

The plants were grown to the flowering stage in a closed greenhouse used for transgenic plant experiments. Chrysanthemum flowers are aggregate, with the ray florets that form the decorative edge containing only female reproductive organs, and tubular florets in the center housing both male and female organs. For crossing experiments, the ray florets were removed before flowering with the heads being bagged. Given the generally low self-pollination rate of chrysanthemum (Wang et al. 2014, Aida et al. 2018), pollinations were performed without emasculation. To monitor selfpollination, three bagged heads within each crossing combination were not artificially pollinated. As 'Sei Arabella' and T37 both have fewer tubular florets than $C$. japonense var. japonense, we used nine chrysanthemum flowerheads and one $C$. japonense var. japonense flowerhead for each set of artificial pollination. Pollen was repeatedly applied to the opened stigma of the seed parent by direct application without using any specialized tool. Seeds were harvested six to eight weeks after pollination. Germination rates were examined on 20 seeds from each cross at seven weeks after sowing. Twelve seedlings were selected from each cross and transplanted into soil for further cultivation in the closed greenhouse.

\section{Polymerase chain reaction (PCR)}

Total DNA was isolated from seedling leaves using the method of Edwards et al. (1991). The primers Cam269F (5'-ctcaaatccaaaagccgecc-3') and Cam1167R (5'-ggaggtttaagggcgtggaa-3') were used to amplify an 899 bp fragment of the Campanula $F^{3} 5^{\prime}{ }^{\prime} H$ gene. DNA quality was checked by amplifying a 549 bp fragment of the endogenous chrysanthemum actin gene using the primers CmActin40F (5'-aatgagcttcgtgtagctcc-3') and CmActin588R (5'-aataccagcagcttccatcc-3'). The DNA was amplified using KOD Plus neo DNA Polymerase (TOYOBO, Osaka, Japan). We performed 32 cycles with 20 seconds at $95^{\circ} \mathrm{C}$, 30 seconds at $62^{\circ} \mathrm{C}$, and one minute at $72^{\circ} \mathrm{C}$.

\section{Characterization of the hybrid plants}

The sizes and shapes of the flowerheads of the hybrids were compared with those of both parents to distinguish hybrid plants from those derived from selfpollination. The colors of the ray florets in the hybrids were visually assessed using the Royal Horticultural Society Colour Charts (RHSCC) as a guide.

\section{Anthocyanin analysis}

High-performance liquid chromatography was performed following the method of Noda et al. (2017). The structures of the major anthocyanins detected in the current study were determined and reported by Noda et al. (2017).

\section{Results and discussion}

\section{Cross fertilization between chrysanthemums and C. japonense var. japonense}

Table 1 lists the seed set rates for all crosses. The rates ranged from $20.6 \%$ to $45.0 \%$ when using 'Sei Arabella' or T37 as the seed parents. Slightly lower but similar rates (from $35.7 \%$ to $53.5 \%$, Aida et al. 2018) were found when using transgenic 'Taihei' in crosses with Chrysanthemum japonense var. japonense. The use of 'Sei Arabella' or T37 as the pollen parents showed higher seed set rates (from $70.7 \%$ to $83.4 \%$ ) than those obtained with 'Taihei' (from $28.8 \%$ to $43.1 \%$, Aida et al. 2018). These results demonstrated that cultivated chrysanthemums, including the color-modified transgenic plants, readily cross with $C$. japonense var. japonense. The seed set rates after crosses with transgenic plants tended to be slightly lower than those from crosses with wild type plants, although in 
(Fig. 1 A). Among the progeny of the transgenic T37 plant (line 2013-03), the transgene was detected in 9 of the 12 plants obtained by crossing 2013-03 as the seed parent and in 10 of the 12 plants obtained by crossing 2013-03 as the pollen parent (Fig. 1 B). The $F 3^{\prime} 5^{\prime} H$ fragment was not amplified from any of the parental wild-type plants (Fig. $1 \mathrm{C}$ ), and the endogenous actin gene was amplified from every $F 3^{\prime} 5^{\prime} H$-negative DNA sample (Fig. 1 A, B, C). These results demonstrated the presence of the transgene in some of the progeny, and further suggested that the PCR-positive plants were hybrids, at least when the pollen parents were transgenic.

\section{Inheritance of modified flower color}

All the progeny plants had flowerhead shapes that were intermediate between those of the parental lines (Fig. 2 and data not shown), suggesting that the plants were hybrids of the two parents, as reported previously (Aida et al. 2018).

Table 2 lists the colors of the ray florets of the hybrids. Among each of the four sets of 12 progeny derived from 'Sei Arabella', 4 or 5 plants were pigmented, and among each of the four sets of 12 T37 progeny, 8 or 9 were pigmented. Therefore, the ratio of plants accumulating anthocyanins was higher in the progeny of T37 than that in the progeny of 'Sei Arabella'. The segregation ratio between pigmented and white was close to 1:3 in the 'Taihei' progeny (Aida et al. 2018); that ratio was lower than those found in the progeny of both 'Sei Arabella' and T37. These differences can be explained by differences in the genetic backgrounds for anthocyanin pigmentation in each of the parental chrysanthemum lines.

All the pigmented progeny of the pink wild-type 'Sei Arabella' and T37 plants had pink petals. When blue transgenic plants were used as the parents, white-, pink-, and blue-petaled plants were produced (Fig. 1 and Table 2). PCR analysis demonstrated that all blue-petaled progeny carried the transgene (Fig. 1), indicating that the transgenes were active in the hybrid progeny. The depths of flower pigmentation in the progeny plants in these experiments were lighter than those of the parental 'Sei Arabella' and T37 lines (Fig. 2); this was also observed in the 'Taihei' progeny (Aida et al. 2018). We assume that hybridization with $C$. japonense var. japonense, which has white flowers, reduced the amounts of anthocyanin

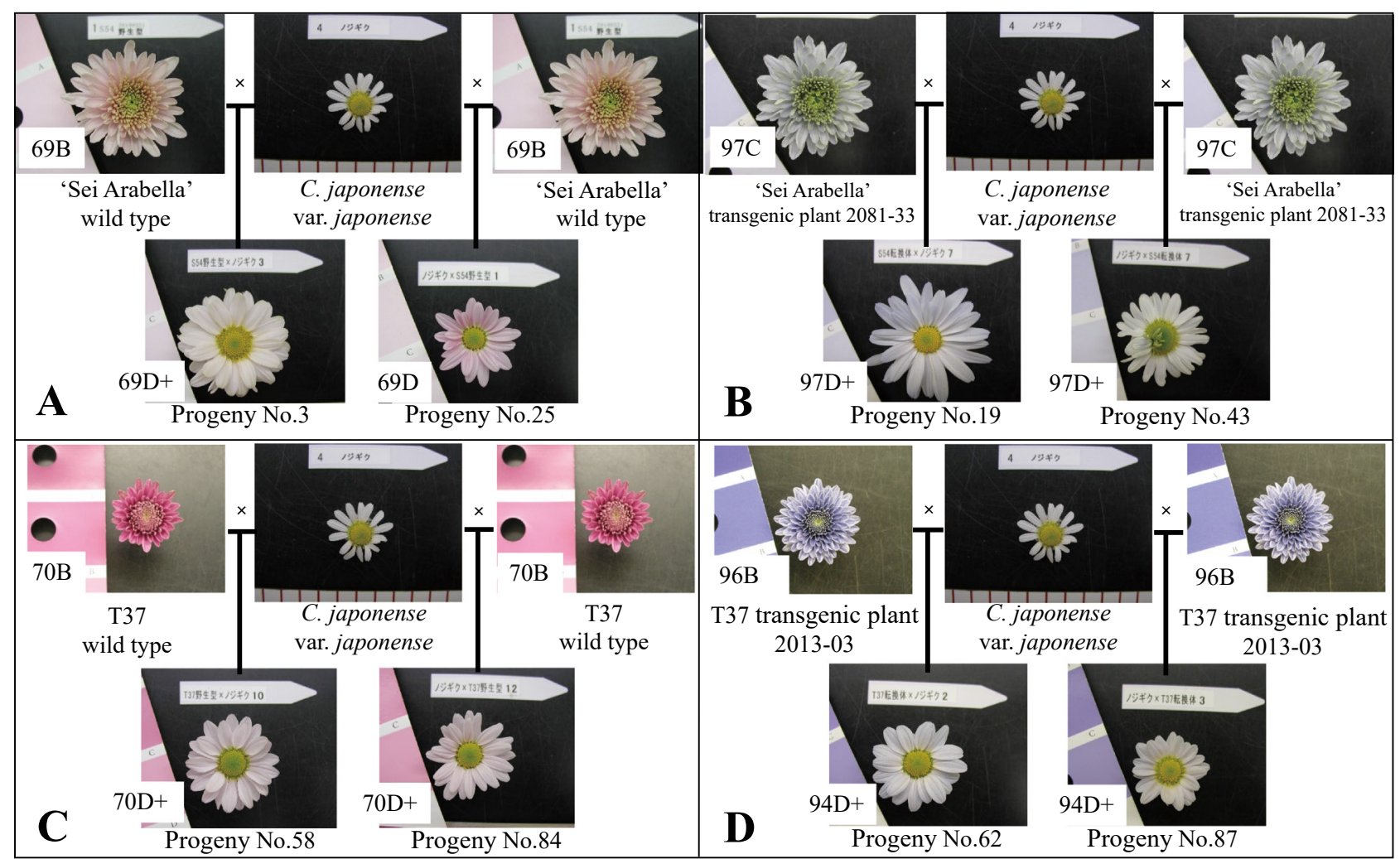

Fig. 2. Modified flower colors of the transgenic plants and their hybrid progeny

The figure shows examples of hybrid plants with flower color hues similar to those of their chrysanthemum parents: 'Sei Arabella' wild type (A), 'Sei Arabella' transgenic plant (B), T37 wild type (C), and T37 transgenic plant (D). Numbers shown at the lower left of each image are the color numbers from the charts of the Royal Horticultural Society; however, many progeny had paler colors (shown as numbers with '+') than the examples shown in the charts. 
precursors, resulting in smaller accumulations of anthocyanins.

Figure 2 shows examples of the inheritance of the modified flower colors by the hybrid plants. Pink progeny were obtained from the pink wild type plants (A: 'Sei Arabella', C: T37) and blue progeny were obtained from the blue transgenic plants (B: 'Sei Arabella' line 2081-33, D: T37 line 2013-03). Based on the RHSCC, parents and progeny appeared to have similar color hues, but were difficult to discern in many progeny due to having colors paler than those shown in the charts. (The pale colors are indicated by color numbers with ' + ' in Fig. 2). Progeny of the T37 transgenic plant showed a slightly purplish hue (94D+) compared with the bluer color of the T37 parent (96B). This result might be caused by the higher accumulation in the progeny than in the parent of a

Table 2. Variations in flower color among hybrid plants

\begin{tabular}{lccccc}
\hline \hline & \multicolumn{5}{c}{ Petal color of ray florets } \\
\cline { 2 - 5 } Crossing combination & & \multicolumn{3}{c}{ Pigmented } \\
\cline { 2 - 5 } & White & Pink & Blue $\begin{array}{c}\text { (Total } \\
\text { pigmented) }\end{array}$ & Total \\
\hline 'Sei Arabella' wild type $\times$ C. japonense var. japonense & 7 & 5 & 0 & $(5)$ & 12 \\
'Sei Arabella' transgenic plant 2081-33 $\times$ C. japonense var. japonense & 8 & 2 & 2 & $(4)$ & 12 \\
T37 wild type $\times$ C. japonense var. japonense & 3 & 9 & 0 & $(9)$ & 12 \\
T37 transgenic plant 2013-03 $\times$ C. japonense var. japonense & 4 & 1 & 7 & $(8)$ & 12 \\
\hline C. japonense var. japonense $\times$ 'Sei Arabella' wild type & 7 & 5 & 0 & $(5)$ & 12 \\
C. japonense var. japonense $\times$ 'Sei Arabella' transgenic plant $2081-33$ & 8 & 2 & 2 & $(4)$ & 12 \\
C. japonense var. japonense $\times$ T37 wild type & 4 & 8 & 0 & $(8)$ & 12 \\
C. japonense var. japonense $\times$ T37 transgenic plant 2013-03 & 3 & 1 & 8 & $(9)$ & 12 \\
\hline
\end{tabular}

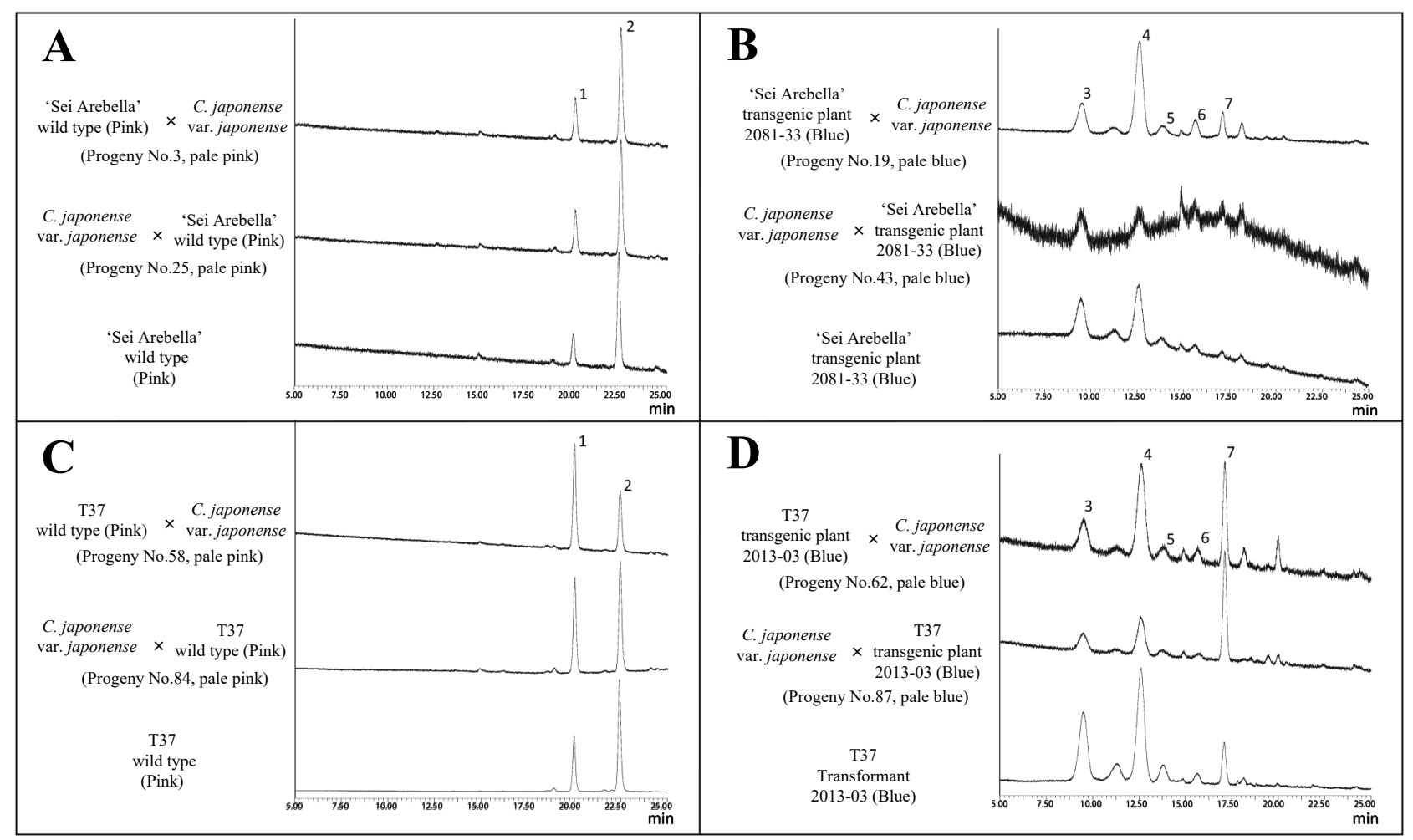

Fig. 3. Anthocyanins detected in the petals of wild-type and transgenic chrysanthemum lines and their hybrid progeny

Shown are representative results from high performance liquid chromatography analyses. The flower colors of the analyzed plants are shown in Figure 2. The progeny line numbers correspond to the results shown in Figures 1 and 2. The wild type plants and their progeny contained the typical chrysanthemum anthocyanins that eluted as peaks 1 and 2 (A, B). The transgenic plants and their progeny contained the modified anthocyanins that eluted as peaks 3 to 7 (C, D). These modified anthocyanins were never detected in wild type plants or their progeny. 
cyanidin-based anthocyanin (peak 7, see Fig. 3) that is purple rather than blue (Noda et al. 2017).

The petals of the progeny contained the same specific anthocyanins as the parents (Fig. 3). The progeny of both the wild type 'Sei Arabella' and T37 plants contained typical chrysanthemum anthocyanins: cyanidin 3-(6"-malonyl)glucoside (peak 1) and cyanidin $3-\left(3^{\prime \prime}, 6^{\prime \prime}\right.$-dimalonyl)glucoside (peak 2). Conversely, the progeny of the blue transgenic plants contained the modified anthocyanins delphinidin 3,3'5'-triglucoside (peak 3), delphinidin 3-(6"-malonyl)glucoside-3',5'-diglucoside (peak 4), delphinidin 3-(3",6"-dimalonyl)glucoside-3',5'diglucoside (peak 5), delphinidin 3-(6"-malonyl)glucoside3'-glucoside (peak 6), and cyanidin 3-(6"-malonyl)glucoside3'-glucoside (peak 7). The modified anthocyanins detected in this study were determined and reported by Noda et al. (2017). The anthocyanins in peaks 3 to 7 were detected only in the transgenic plants. These results demonstrated that the transgenes were both inherited and functional in the hybrid progeny.

Figure 1 shows that some transgene-carrying progeny of 'Sei Arabella' and T37 had white flowers, as also found among progeny of transgenic 'Taihei' plants (Aida et al. 2018). These white-flowered plants may lack functional alleles of genes needed for anthocyanin production. Therefore, it will be difficult to identify transgene-bearing plants by flower color alone. The latent transgenic phenotype would reappear after recrossing with chrysanthemums that carry functional anthocyanin biosynthesis genes.

\section{Future commercialization of blue transgenic chrysanthemums}

Chrysanthemum cultivars can cross with wild Chrysanthemum species (Nakata et al. 1987) and some naturally resulting hybrids have been classified as independent species (Ohashi \& Yonekura 2004). Indeed, the naturally crossed hybrids of Chrysanthemum wakasaense and a chrysanthemum cultivar survived in the wild for more than 14 years (Nakata et al. 2001). In this study and our previous study (Aida et al. 2018), we demonstrated the crossability of blue transgenic chrysanthemums with the wild species $C$. japonense var. japonense. We also demonstrated inheritance of the transgenes and modified flower color in hybrid progeny. These results suggest a possibly high risk of transgenes escaping from cultivated chrysanthemums to wild Chrysanthemum populations. For the future commercialization of blue chrysanthemums, we are investigating techniques to reduce the possibility of transgene transfer to wild species.

\section{Acknowledgments}

We wish to thank Inochio Seikoen Ltd. for providing the chrysanthemum cultivar 'Sei Arabella' and the breeding line T37. We would also like to thank the Aomori Prefectural Industrial Technology Research Center for providing the Clitoria ternatea $A 3^{\prime} 5^{\prime} G T$ gene and the chrysanthemum $F 3 H$ promoter, and Dr. K. Kato (Nara Institute of Science and Technology) for providing the tobacco $A D H-5$-UTR region and the Arabidopsis $H S P$ terminator; all of which are components of the binary vector $\mathrm{pB} 423$. This work was supported in part by a Cooperative Research Grant from the Gene Research Center, University of Tsukuba.

\section{References}

Aida, R. et al. (2018) Inheritance of bluish flower color of transgenic chrysanthemum by interspecific hybrids. $J A R Q$, 52, 339-345.

Brugliera, F. et al. (2013) Violet/blue chrysanthemumsMetabolic engineering of the anthocyanin biosynthetic pathway results in novel petal colors. Plant Cell Physiol., 54, 1696-1710.

Edwards, K. et al. (1991) A simple and rapid method for the preparation of plant genomic DNA for PCR analysis. Nucl. Acids Res., 19, 1349.

Katsumoto, Y. et al. (2007) Engineering of the rose flavonoid biosynthetic pathway successfully generated blue-hued flowers accumulating delphinidin. Plant Cell Physiol., 48, 1589-1600.

Nakata, M. et al. (1987) Species of wild Chrysanthemums in Japan: cytological and cytogenetical view on its entity. Acta Phytotax. Geobot., 38, 241-259 [In Japanese with English summary].

Nakata, M. et al. (2001) A follow-up survey on the population of Dendranthema japonicum var. wakasaense where the hybrids with garden chrysanthemum were discovered 14 years ago. Jpn. J. Conserv. Ecol., 6, 21-27 [In Japanese].

Noda, N. et al. (2013) Genetic engineering of novel bluercolored chrysanthemums produced by accumulation of delphinidin-based anthocyanins. Plant Cell Physiol., 54, 1684-1695.

Noda, N. et al. (2017) Generation of blue chrysanthemums by anthocyanin B-ring hydroxylation and glucosylation and its coloration mechanism. Sci. Adv., 3, e160278.

Ohashi, H. \& Yonekura, K. (2004) New combination in Chrysanthemum (Compositae-Anthemideae) of Asia with a list of Japanese species. J. Jpn. Bot., 79, 186-195.

Tanaka, Y. et al. (1998) Metabolic engineering to modify flower color. Plant Cell Physiol., 39, 1119-1126.

Wang, F. et al. (2014) Identification of chrysanthemum (Chrysanthemum morifolium) self-incompatibility. Sci. World J., 2014, 625658. 